\title{
Surgical Robotics Systems for Deep Brain Neurosurgery
}

\author{
Andrew M. K. Nassief ${ }^{1}$
}

${ }^{1}$ Affiliation not available

January 11, 2022

\begin{abstract}
Robotics systems designed for surgical applications such as Neurosurgery, likely may need to implement synchronous communication in real time and asynchronous learning. It will likely primarily be oriented towards spatial imaging and 3D virtualization, various communication protocols, and calibration settings in order to perform optimal results. In regards to computation, it needs to be heavily fault tolerant in operation. It also needs to be aware of false positives. Likely a complex deep brain surgical robotics system would implement variations of brain mapping technology and may utilize topological neuroanatomy. Various technologies in regards to the mapping of the brain, visualization, robotics and mechatronics systems would need to be in place. This paper is to look at the sciences through a theoretical and conceptual process. This isn't FDA reviewed for medical accuracy and is meant to warrant a theoretical paper where information is "as-is". This will hopefully provide a blueprint for continuing research later on.
\end{abstract}
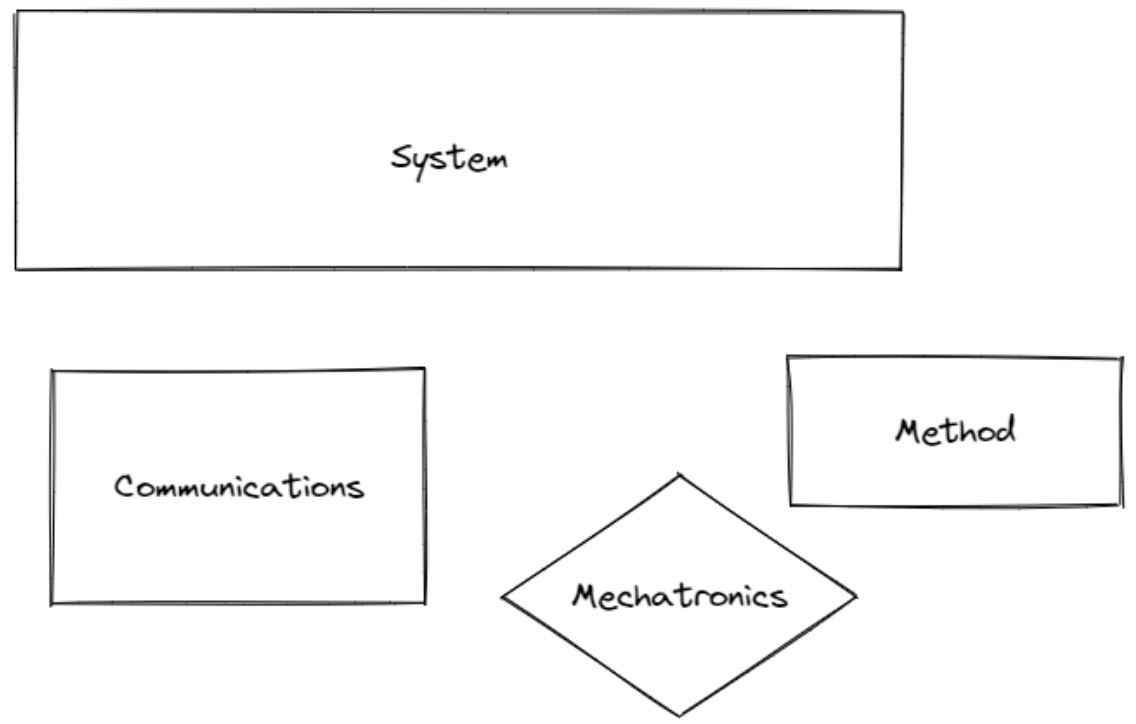

Figure 1: This is caption

In order for a surgical system to be efficient enough to perform an operation, one needs to keep in mind how the core of said system is developed. Fault tolerance would be key to such a system. Fault tolerance would involve making sure such a system would have some sort of a backup in response to a hardware or software issue or failure. In a hospital setting, many things in relation to fault tolerance need to be considered. This 
goes well beyond standard backups for power in event of a power outage, but also hard coded fault tolerant mechanisms and backup hardware.

In relation to computation, cellular automata will play a large role in regards to space vectorization and surface area recognition. Computationally, a said program can only know to analyze what it can understand. You have different variations of chaotic and complex CML systems when dealing with the brain. Various layers and components of the brain also have different layers and degrees of freedom. This is important given the regard to how accurate such a system needs to be.

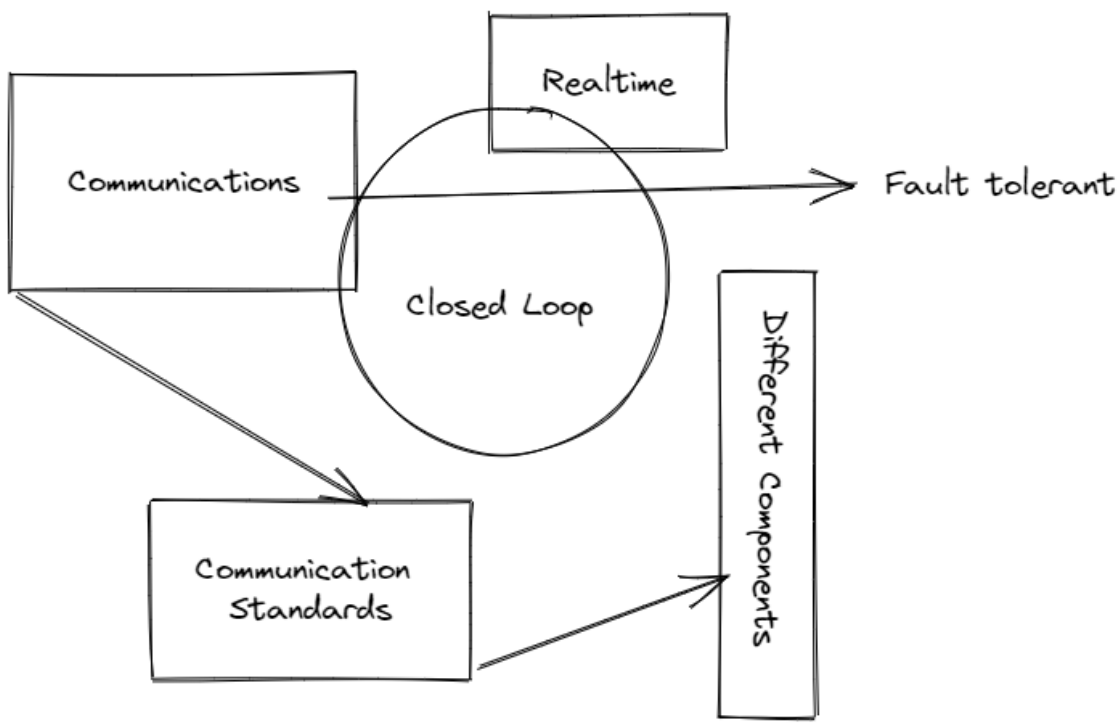

Figure 2: This is a caption

Communication is going to be essentially very crucial. This is especially true given that many surgical procedures are time sensitive. That means an optimization of time is critical, how different components and systems interact with each other is crucial, and that said system needs to be fault tolerant. It would also implement a closed-loop type system where everything was preconfigured as a result of a learning model in regards to said optimal result.

Something crucial to understand is that the learning mechanism will likely need to implement multiple components. When dealing with a high level and degree of sensitivity such as the brain, preconfigured imaging of the brain is likely a must. It is also important to consider the lateralization of the brain, size, and hemisphere. Size variation and difference are also important to consider. This is why there is both a computer vision aspect to it, as well as preconfigured modeling in regards to degrees of freedom and spatial density. 


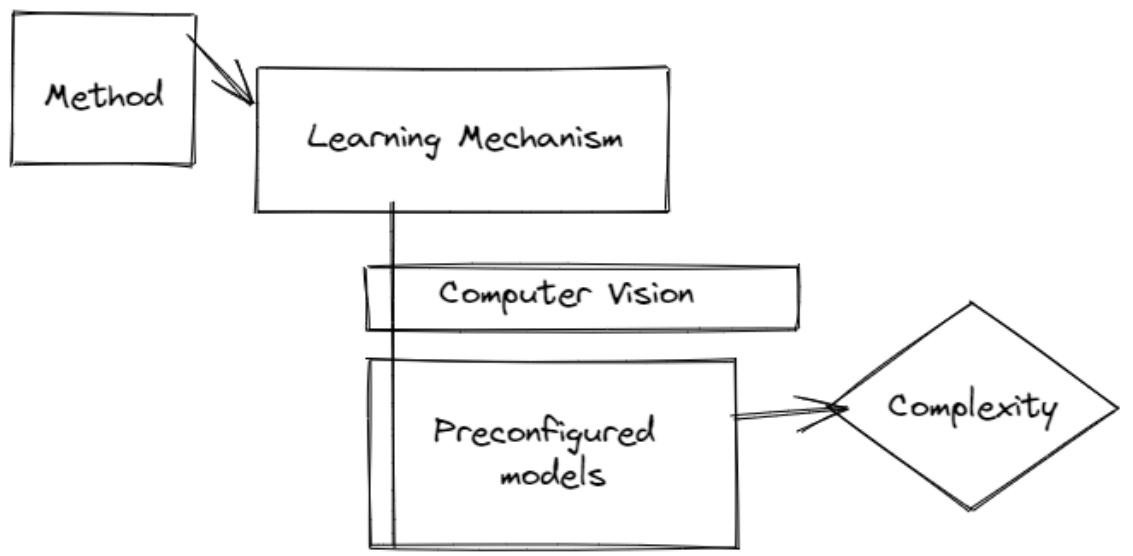

Figure 3: This is a caption

A question one might consider, is what is complexity? There are different degrees of complexity in regards to a surgical robotics system, especially one that targets the brain. This can be something as simple as time complexity, but in regards to the focus being learning methods, a primary concern is complexity of the brain. Degrees of freedom, spatial geometry, topology, and density or composition are all crucial learning considerations.

These different considerations in regards to communication, complexity and fault tolerance serve as a basic introduction of this idea. The research regarding deep brain neurosurgery and robotics is currently emerging and being developed throughout different fields. Therefore, likely the author will be publishing further research or innovations related to this subject matter. In regards to how such a system is, the components and how they communicate will be key, as well as the system as a whole. 